\title{
O desenvolvimento profissional dos alunos de Pedagogia: Nível de aquisição de competências
}

\author{
Sonia Casillas Martín ${ }^{l}$ \\ Marcos Cabezas González \\ Sara Serrate González \\ Universidad de Salamanca, Salamanca, España
}

\section{Resumo}

Este artigo expõe os resultados de um estudo centrado no desenvolvimento de competências práticas autopercebidas pelos estudantes de Pedagogia. O objetivo é saber se o nível de autopercepção na aquisição de competências gerais e específicas para o exercício da profissão pedagógica varia após a realização das práticas e verificar a existência de diferenças significativas na autopercepção do aluno de acordo com as variáveis: ano de conclusão dos estudos, desempenho acadêmico, especialização matriculada e tipo de instituição de estágio. A metodologia empregada é quantitativa, o desenho de investigação de tipo pre-experimental, com aplicação de pré-teste e pósteste. Utilizou-se um método descritivo-inferencial, por meio do estudo de pesquisas eletrônicas de opinião. Os resultados mostram que a valoração dos futuros pedagogos sobre a aquisição de diferentes competências do curso de Pedagogia, é muito positiva antes e depois do período de práticas externas; assim como a existência de diferenças significativas em função das variáveis estudadas.

Palavras-chave: práticas profissionais, competência profissional, ensino superior.

Abstract: The professional development of Pedagogy students: Level of acquired competences

This article presents the results of a study focused on the development of self - perceived practical skills by Pedagogy majors. The objective is to know if the level of self-perception in the acquisition of general and specific competences for the exercise of the pedagogical profession varies after the accomplishment of the teacher training period and verify the existence of significant differences in the student's self-perception according to the variables: year of studies completion, academic performance, specialization enrollment and type of internship institution. The methodology used is quantitative, the research design is of the pre-experimental type, with application of pre-test and post-test. A descriptive-inferential method was used, through the study of electronic opinion surveys. The results reveal that the future teachers' evaluation of the acquisition of different competences in the Degree Program in Pedagogy is very positive, both before and after the period of external teaching training; as well as the existence of significant differences according to the studied variables.

Keywords: professional practices, professional competence, higher education.

Resumen: El desarrollo profesional de los estudiantes de Pedagogía: Nivel de adquisición de competencias

Este artículo expone los resultados de un estudio centrado en el desarrollo de competencias prácticas autopercibidas por los estudiantes de pedagogía. El objetivo es conocer si el nivel de autopercepción en la adquisición de competencias generales y específicas para el ejercicio de la profesión pedagógica, varía tras la realización de las prácticas y verificar la existencia de diferencias significativas en la autopercepción del estudiante en función de las variables: año de finalización de estudios, rendimiento académico, mención cursada y titularidad del centro de prácticas. La metodología utilizada es cuantitativa, el diseño de investigación de tipo pre-experimental, con aplicación de pre-test y post-test. Se empleó un método descriptivo-inferencial, por medio del estudio de encuesta electrónica. Los resultados muestran que la valoración de los futuros pedagogos sobre la adquisición de diferentes competencias del Grado de Pedagogía, es muy positiva tanto antes como después del periodo de prácticas externas; así como la existencia de diferencias significativas en función de las variables estudiadas.

Palabras clave: prácticas profesionales, competencia profesional, enseñanza superior.

Endereço para correspondência: Universidad de Salamanca. Salamanca. España. E-mail: scasillasma@usal.es. Facultad de Educación. Paseo de Canalejas, 169, 37008, Salamanca.

Telefone: +34 663165035 - Fax: +34 923294693 


\section{Introducción}

El entrenamiento profesional en la formación inicial de los pedagogos continúa siendo una preocupación constante en la comunidad científica (Latorre \& Blanco, 2011). La implantación del Espacio Europeo de Educación Superior (EEES) ha promovido una nueva concepción, existente ya en diferentes contextos profesionales, del proceso de enseñanza-aprendizaje y del desarrollo profesional: el trabajo por competencias (Vilà, Aneas, \&Rajadell, 2015). En la Universidad, las competencias adquieren un matiz claramente profesionalizador, ya que el universitario, como futuro profesional, podrá ocupar un lugar en un mercado laboral especializado en el que deberá desenvolverse de manera competente (Armengol, Castro, Jariot, Massot, \& Sala, 2011).

Las competencias son "el conjunto de conocimientos,procedimientos $\mathrm{y}$ actitudes que se complementan entre sí, y que capacitanpara actuar con eficacia en las diferentes situaciones profesionales, aportandoun saber (conocimientos), un saber hacer (destrezas) y un saber estar (actitudes) en cada actuación" (Armengol et al., 2011, p. 77); y su desarrollo real depende de las oportunidades para poder ponerlas en práctica (Aneas \&Vilà, 2015). Por ello, con la implantación del EEES, el Prácticum nace como un escenario ideal tanto para adquirir y poner en práctica competencias profesionales como para facilitar el tránsito entre el mundo académico y el laboral (Mendoza \& Covarrubias, 2014).

La formación práctica del alumnado constituye uno de los ámbitos más relevantes en los nuevos Planes de Estudio de las diferentes titulaciones (González \& Fuertes, 2011). El Prácticum es una modalidad de prácticas externas. De Miguel (2005) identifica tres modalidades al respecto: el Prácticum, materia con un número de créditos determinado que forma parte del plan de estudios de una titulación universitaria; las prácticas en empresa, que no forma parte de un plan de estudios y que son realizadas por alumnos titulados; y las prácticas clínicas, propias del área de las ciencias de la salud.En la actualidad, el Prácticum es una materia destinada a enriquecer la formación inicial, complementando los aprendizajes académicos con la experiencia en centros de trabajo (Cid, Pérez, \& Sarmiento, 2011) y tiene un marcado carácter profesionalizador (Rial \&Barreira, 2012). Rodríguez, Calmaestra y Maestre (2015), conciben el Prácticum como

un periodo formativo en el que se articula la acción de la Universidad y de otros centros profesionales externos para ofrecer al estudiante una tutorización sobre su fase de prácticas. Ésta se desarrolla en un contexto profesional real en la cual dicho estudiante debe participar activamente y conectar teoría y práctica a través de la reflexión en la acción para poder adquirir e interiorizar los conocimientos, destrezas, habilidades y actitudes propias de la profesión para la que se está formando (p.415).

Tiene un enfoque más curricular e integrado de los estudios, que nos sitúa en un proyecto formativo más completo en el que la unidad es el propio plan de estudios. Así, el Prácticumse propone alcanzar el perfil profesional al que pertenece, desde la integración y la función formativa(Zabalza, 2011; Latorre \& Blanco, 2011) y pretende conseguir los objetivos de: (1) tomar conciencia sobre la cultura profesional; (2) conocer contextos de referencia que permitan comprender los acontecimientos y fenómenos que tienen lugar en la realidad profesional; (3) poner en práctica experiencias formativas diferentes de las lectivas de aula; (4) crear una identidad profesional individual y colectiva; y (5) construir estrategias para acceder al mundo del empleo (Aneas, Vilà, \&Alòs, 2015).

El Prácticum en pedagogía es una materia universitaria que pretende completar la formación de los futuros pedagogos mediante su inmersión temporal en un contexto profesional vinculado con el mundo de la educación (Rego, Rial\&Barreira, 2015). En los planes de estudio conducentes al Grado en Pedagogía, esta disciplina constituye una actividad formativa realizada por los estudiantes y supervisada por las Universidades, cuyo objetivo es permitir a los mismos aplicar y complementar los conocimientos adquiridos en su formación académica, favoreciendo la adquisición de competencias que les preparen para el ejercicio de actividades profesionales, faciliten su empleabilidad y fomenten su capacidad de emprendimiento (RD 1707/2011).

En el caso de la Facultad de Educación de la Universidad de Salamanca (España), se desarrolla durante elcuarto curso con una carga de 24 European Credit Transfer and Accummulation System (ECTS) (240 horas). Este periodo formativo, con entidad propia, supone la estancia en la institución de prácticas y la asistencia a una serie de seminarios en la Universidadque permiten y favorecen la reflexión sobre la propia práctica. Para la realización de las mismas se contemplan dos menciones: una dedicada específicamente a la Formación y Gestión de calidad y la otra enfocada a la Orientación Educativa y el Asesoramiento; existe, no obstante, una tercera opción denominada sin mención para aquellos casos en los que el alumnado se encuentra matriculado de asignaturas propias de alguna de las dos menciones y de otras libres que no son conducentes a una mención específica (Serrate, Casillas,\& Cabezas, 2016).Vinculada con la mención que se encuentran cursando los estudiantes, los centros en los que pueden realizar prácticas se organizan en tres bloques en función de la tipología: ámbito de la orientación escolar (departamentos o equipos de orientación, educativa, centros, centros de Educación Infantil y Primaria), ámbito de la formación y gestióneducativa (servicios especializados 
en formación educativa, empresas, organismos de gestión educativa), y un tercer ámbito denominado social y que se organiza en función de los destinatarios con los que trabajan las instituciones de acogida de los estudiantes (centros de atención a personas con discapacidad, centros de protección de menores, tercera edad).

Son diversas las investigaciones cuyo objeto de estudio se centra en diferentes dimensiones del Prácticum de las titulaciones de Educación (Armengol et al.,2011; Bretones, 2013; Cabrerizo, Rubio, \& Castillo, 2010; Cabrerizo, Rubio, Castillo, \&Cabrerizo, 2011; Casillas, González, \& Serrate, 2015; González, 2006; Latorre \& Blanco, 2009; Rodicio\& Iglesias, 2011; Serrate et al.,2016). En este trabajo nos centraremos en el nivel de adquisición y de desarrollo de competencias generales (CG) y específicas (CE)autopercibidas por los futuros pedagogos para el ejercicio de su profesión durante el periodo de prácticas externas.

\section{Hipótesis y objetivos}

En este trabajo se contempla una hipótesis principal: la autopercepción del alumnado respecto a la adquisición de competencias generales y específicas en la materia prácticas externas aumenta tras la realización de las prácticas.

El objetivo fundamental es el de conocer el nivel de adquisición de competencias generales y específicas para el ejercicio de la profesión pedagógica manifestado antes y después de la realización de las prácticas, por los estudiantes que finalizaron sus estudios en los cursos académicos 2014-2015 y 2015-2016.

Partiendo de las sugerencias de mejora propuestas por los alumnos del curso 2014-2015, relacionadas fundamentalmente con las instituciones colaboradoras y receptoras de alumnos en prácticas, con el proceso de adjudicación del estudiante al centro de prácticas y con los materiales de evaluación; pretendemos comprobar si los ajustes y cambios realizados en el Prácticum del curso académico 2015-2016, originan diferencias significativas en la percepción manifestada por los estudiantes de este último curso. Esta comparación permitirá verificar si estos cambios han mejorado la gestión de las prácticas.

Del mismo modo se pretende comprobar la existencia de diferencias, en esta percepción, en función del rendimiento académico de los alumnos, la mención cursada y la titularidad del centro en el que realizaron las prácticas.

\section{Método}

Con el fin de concretar las valoraciones de sus propios protagonistas, cuestión relevante para la mejora y calidad de este proceso de formación profesional, se ha optado por utilizar una metodología cuantitativa, con un diseño de investigación de tipo pre-experimental con pretest y pos-test (Campbell \&Standley, 1963).Se utilizó un método descriptivo-inferencial, por medio del estudio de encuesta electrónica aplicada antes de comenzar y una vez finalizada la estancia de prácticas en el centro.

Este tipo de diseño pre-post nos permite conocer el nivel de competencia autopercibida que posee el estudiante antes de comenzar su formación práctica y cuál es el nivel autopercibido una vez finalizada la misma, para poder verificar si esta formación es adecuada para adquirir las competencias evaluadas.

\section{Población y Muestra}

La población objeto de estudio está formada por un total de 120 alumnos que, durante los cursos 20142015 y 2015-2016, cursaron los 24 créditos (240 horas) de la asignatura de Prácticum del Grado de Pedagogía en la Universidad de Salamanca (España). La muestra final, compuesta por 99 estudiantes, supone un $\pm 4,1 \%$ de error muestral para un nivel de confianza del $95 \%$, lo que informa suficientemente de su representatividad.

En relación a la composición de la muestra, como es habitual en titulaciones propias del campo de las Ciencias de la Educación, es mayor el número de mujeres que el de hombres( $82 \%$ y $18 \%$ respectivamente). El Grado de Pedagogía de la Universidad de Salamanca cuenta con tres itinerarios formativos, planteados con carácter de mención: (1) Formación y gestión de calidad, (2) Orientación educativa y asesoramiento y (3) mención libre. El $54 \%$ se encuentran cursando la mención de Orientación Educativa y asesoramiento y el $74,6 \%$ han realizado las prácticas en un centro público (titularidad pública) o concertado (titularidad privada).

\section{Instrumento}

Es de naturaleza cuantitativa, basado en la metodología de encuesta estructurada, dentro de la que se incluyó una escala tipo Likert de 5 puntoscon valores que oscilan entre nada (1) y mucho (5) para la escala de competencias profesionales, en ambas aplicaciones. El cuestionario diseñado ad hoc por el equipo de coordinación del Prácticum, consta de 27 ítems incluidos en tres bloques de contenidos: 4 ítems constituyen los datos sociodemográficos (sexo, curso,mención y titularidad del centro de prácticas), y 23 ítems corresponden a las competencias profesionales del Pedagogo, 6 de ellas generales y 17 específicas. Cada uno de los ítems mide el nivel autopercibido de adquisición de las competencias,y para ello se pidió a los estudiantes que,en función de sus expectativas, valorasen en qué medida pensaban que iban a adquirir las competencias planteadas antes de realizar las prácticas 
(aplicación pre-test) y posteriormente,tras su periodo de prácticas externas, se les solicitó que valorasen en qué medida consideran haber adquirido las competencias descritas (aplicación pos-test).

Los ítems recogen las competencias que se presentan en la siguiente tabla:
La validez de contenido fue garantizada por medio de la valoración de jueces expertos, académicos especializados tanto en metodología como en el tema de investigación objeto de estudio $\mathrm{y}$, algunos de ellos, con amplia y reconocida experiencia en la coordinación, organización y gestión de la materia de Prácticum. Respecto

Tabla 1

Competencias Prácticum de Pedagogía

Generales

CG1. Capacidad para el trabajo en equipo

CG2. Capacidad de aprendizaje autónomo y responsabilidad

CG3. Capacidad creativa y emprendedora, actitud innovadora y de adaptación al cambio

CG4. Capacidad para valorar el impacto social y medioambiental de actuaciones y decisiones en el ámbito educativo y social

CG5. Capacidad de crítica y autocrítica, de toma de conciencia y de adopción de actitudes vinculadas a concepciones éticas y deontológicas

CG6. Capacidad de autoconocimiento para el desarrollo personal y profesional

Específicas

CE1. Diagnosticar las necesidades y posibilidades de desarrollo de las personas

CE2. Conocer y comprender los elementos, procesos y valores de educación y su incidencia en la formación integral

CE3. Diseñar planes, programas, proyectos, propuestas innovadoras de formación, acciones y recursos adaptados a los distintos niveles del sistema educativo

CE4. Evaluar planes, programas, proyectos, propuestas innovadoras de formación

CE5. Desarrollar estrategias y técnicas para promover la participación y el aprendizaje a lo largo de la vida

CE6. Aplicar y coordinar programas educativos de desarrollo personal, social y profesional

CE7. Desarrollar y coordinar intervenciones educativas con personas o grupos, con necesidades específicas, en situaciones de riesgo, de desigualdad o discriminación

CE8. Conocer y evaluar políticas, instituciones sistemas y organismos educativos

CE9. Conocimiento y aplicación de las herramientas propias del diagnóstico, evaluación y análisis en Pedagogía

CE10. Organizar y gestionar centros, instituciones, servicios y recursos educativos y formativos

CE11. Supervisar planes, programas, centros y profesionales de la educación y la formación

CE12. Desarrollar procesos y modelos de gestión de calidad de la educación y la formación

CE13. Aplicar estrategias y técnicas de tutorización, entrenamiento, asesoramiento entre iguales, consulta y orientación en procesos educativos y formativos

CE14. Asesorar sobre el uso pedagógico e integración curricular de los medios didácticos

CE15. Analizar, diseñar y evaluar las aplicaciones de las tecnologías de la información y la comunicación

CE16. Identificar planteamientos y problemas educativos, indagar sobre ellos

CE17. Diagnóstico de situaciones complejas con especial atención a la diversidad y a la inclusión social

CE18. Desarrollar procesos y modelos de gestión de calidad de la educación y la formación

CE19. Aplicar estrategias y técnicas de tutorización, entrenamiento, asesoramiento entre iguales, consulta y orientación en procesos educativos y formativos

CE20. Asesorar sobre el uso pedagógico e integración curricular de los medios didácticos

CE21. Analizar, diseñar y evaluar las aplicaciones de las tecnologías de la información y la comunicación

CE22. Identificar planteamientos y problemas educativos, indagar sobre ellos

CE23. Diagnóstico de situaciones complejas con especial atención a la diversidad y a la inclusión social 
a la consistencia interna del instrumento, la correlación inter-elementos promedio, arroja un coeficiente Alfa de Cronbach de 0,916 en su aplicación pre y de 0,924 en su aplicación post.

\section{Procedimiento}

Para realizar el estudio se solicitó a los estudiantes que cumplimentaran el cuestionario antes de comenzar y al finalizar el periodo de prácticas externas. Este período abarca desde mediados de noviembre hasta mediados de febrero, con una duración de 3 meses, en los que tienen que realizar 240 horas de trabajo práctico.Al instrumento, diseñado por medio del servicio de diseño y aplicación en formato electrónico (Díaz, 2012), accedían los estudiantes a través de una plataforma virtual de tipo Moodle.

\section{Análisis de los datos}

Se utilizó el programa informático de análisis estadístico SPSS v.21. El tratamiento de los mismos se centró en un estudio descriptivo, por medio del análisis de medias sobre las percepciones que tenían los estudiantes antes de comenzar las prácticas, así como las medias sobre la percepción al finalizar dicho periodo.También se realizó un análisis de tipo inferencial a partir de una comparación de medias. Una vez comprobados los supuestos paramétricos de normalidad (prueba de Kolmogorov-Smirnov y Shapiro-Wilk) y de Homocedasticidad (prueba de Levene), se optó por la utilización de pruebas paramétricas, de contraste de hipótesis, en concreto la prueba de $\mathrm{T}$ de student para muestras independientes y para muestras relacionadas, además de la Prueba ANOVA; ambas permitieron comprobar la existencia de diferencias estadísticamente significativas en cuanto a las valoraciones recogidas en las dos aplicaciones (pre y post). Del mismo modo se comprobó la existencia de diferencias en función del curso académico en el que los estudiantes finalizaron el Grado, del rendimiento académico, de la mención cursada yde la titularidad del centro (público o concertado).Para el estudio del rendimiento académico, se tendrá en cuenta la nota final que obtiene el estudiante y que queda recogida en las actas de evaluación de la asignatura del Prácticum.

\section{Resultados}

A continuación, exponemos los principales resultados hallados en esta investigación.

\section{Diferencias generales en función de la aplicación (pre vs post)}

En general, la valoraciónde los estudiantes sobre la adquisición de las diferentes competencias del Grado de
Pedagogía que se proponen es muy positiva tanto en la aplicación pre-test como en el post-test(tabla 2). Aunque lo deseable hubiera sido lo contrario, los alumnos otorgan puntuaciones más elevadas en muchas de las competencias evaluadas.

Se evidencian diferencias significativas en cuatro de las veintitrés competencias examinadas en la aplicación pre-test, vinculadas fundamentalmente a la capacidad emprendedora de los estudiantes (CG3), al diagnóstico de necesidades y posibilidades de desarrollo (CE1), a la evaluación de planes, programas y proyectos (CE4) y al desarrollo de estrategias que promuevan la participación (CE5). Respecto a la aplicación post-test, existen diferencias significativas en tres de las competencias, vinculadas a la capacidad para valorar el impacto social y medioambiental de las actuaciones como pedagogos (CG4), a la capacidad de conocer y comprender los elementos y procesos educativos (CE2) y a la organización y gestión de centros, instituciones o servicios (CE10).

\section{Diferencias en las valoraciones en función del curso académico (2014-2015/2015-2016)}

En el Sistema Universitario español, curso académico se refiere al periodo del año en que los estudiantes universitarios realizan sus estudios. El Grado en Pedagogía está organizado en cuatro cursos académicos. La materia de Prácticum se imparte en el cuarto curso.

Los estudiantes de la segunda promoción examinada otorgan, en total, puntuaciones más altas a la adquisición de competencias respecto a la primera promoción $\left(\mu_{p r o-}\right.$ moción 1 4,08; $\left.\mu_{\text {promoción } 2}=4,65\right)$. A partir de la exploración en función del curso académico,como puede verse en la tabla 3, encontramos en la primera promoción examinada diferencias significativas en doce de las veintitrés competencias, todas ellas en la aplicación post-test. En su mayoría existe coincidencia con la segunda promoción examinada (estudiantes que finalizan sus estudios en el curso 2015-2016), sin embargo tan solo se encuentran, en esta segunda promoción, diferencias en la adquisición de una de las competencias: Desarrollar y coordinar intervenciones educativas con personas o grupos, con necesidades específicas, en situaciones de riesgo, de desigualdad o discriminación (CE7) (Pre-test $\mu_{\text {promoción } 1}=3.83$; $\mu_{\text {promoción } 2}=3.67 ;$ Pos-test $\mu_{\text {promoción1 }}=4.10 ; \mu_{\text {promoción } 2}=3.90$ ) en la aplicación post-test y en una de las competencias en la aplicación pre-test, vinculada a la identificación de planteamientos y problemas educativos (CE22) (Pre-test $\mu_{\text {promoción } 1}=3.43 ; \mu_{\text {promoción } 2}=3.96 ;$ Pos-test $\mu_{\text {promoción } 1}=3.90$; $\mu_{\text {promoción } 2}=3.73$ ).

\section{Diferencias en las valoraciones en función del rendimiento académico}

En este apartado se pretende comprobar si el rendimiento académico de los estudiantes influye en 
Tabla 2

Diferenciade mediasen función de la aplicación

\begin{tabular}{llllllll}
\hline Competencias & \multicolumn{1}{l}{ Pre Test } & \multicolumn{3}{l}{ Post Test } & & \\
\hline Generales & $\mu$ & $\sigma$ & $\mu$ & $\sigma$ & $t$ & gl & $p$ \\
\hline CG1. Capacidad para el trabajo en equipo & 3,97 & 0,77 & 4,07 & 0,89 & $-1,06$ & 98 & 0,28 \\
CG2. Capacidad de aprendizaje autónomo y responsabilidad & 4,30 & 0,76 & 4,32 & 0,80 & $-0,20$ & 98 & 0,83 \\
$\begin{array}{l}\text { CG3. Capacidad creativa y emprendedora, actitud innovadora y de } \\
\text { adaptación al cambio }\end{array}$ & 3,98 & 0,79 & 3,78 & 1,01 & 1,91 & 98 & 0,05 \\
$\begin{array}{l}\text { CG4. Capacidad para valorar el impacto social y medioambiental de } \\
\text { actuaciones y decisiones en el ámbito educativo y social }\end{array}$ & 3,51 & 1,04 & 3,87 & 0,85 & $-3,10$ & 98 & 0,00 \\
$\begin{array}{l}\text { CG5. Capacidad de crítica y autocrítica, de toma de conciencia y de } \\
\text { adopción de actitudes vinculadas a concepciones éticas y deontológicas }\end{array}$ & 4,15 & 0,82 & 4,24 & 0,68 & $-0,98$ & 98 & 0,32 \\
$\begin{array}{l}\text { CG6. Capacidad de autoconocimiento para el desarrollo personal y } \\
\text { profesional }\end{array}$ & 4,20 & 0,75 & 4,32 & 0,71 & $-1,48$ & 98 & 0,14
\end{tabular}

Específicas

CE1. Diagnosticar las necesidades y posibilidades de desarrollo de las personas

CE2. Conocer y comprender los elementos, procesos y valores de educación y su incidencia en la formación integral

CE3. Diseñar planes, programas, proyectos, propuestas innovadoras

de formación, acciones y recursos adaptados a los distintos niveles del sistema educativo

CE4. Evaluar planes, programas, proyectos, propuestas innovadoras de formación

CE5. Desarrollar estrategias y técnicas para promover la participación y el aprendizaje a lo largo de la vida

CE6. Aplicar y coordinar programas educativos de desarrollo personal, social y profesional

CE7. Desarrollar y coordinar intervenciones educativas con personas

o grupos, con necesidades específicas, en situaciones de riesgo, de

desigualdad o discriminación

CE8. Conocer y evaluar políticas, instituciones sistemas y organismos educativos

CE9. Conocimiento y aplicación de las herramientas propias del

diagnóstico, evaluación y análisis en Pedagogía

\begin{tabular}{lllllll}
$\mu$ & $\sigma$ & $\mu$ & $\sigma$ & $t$ & $\mathrm{gl}$ & $p$ \\
\hline 4,00 & 0,99 & 3,79 & 1,07 & 2,02 & 98 & 0,04
\end{tabular}

$\begin{array}{lllllll}3,88 & 0,84 & 4,11 & 0,80 & -2,22 & 98 & 0,02\end{array}$

$\begin{array}{lllllll}3,48 & 1,04 & 3,45 & 1,18 & 0,25 & 98 & 0,79\end{array}$

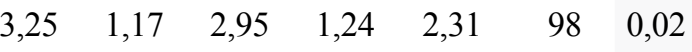

$\begin{array}{lllllll}3,65 & 0,84 & 3,38 & 1,10 & 2,24 & 98 & 0,02\end{array}$

$\begin{array}{lllllll}3,54 & 0,94 & 3,32 & 1,25 & 1,61 & 98 & 0,10\end{array}$

$\begin{array}{lllllll}3,99 & 1,02 & 3,93 & 1,28 & 0,51 & 98 & 0,60\end{array}$

CE10. Organizar y gestionar centros, instituciones, servicios y recursos educativos y formativos

CE11. Supervisar planes, programas, centros y profesionales de la educación y la formación

CE12. Desarrollar procesos y modelos de gestión de calidad de la

educación y la formación

CE13. Aplicar estrategias y técnicas de tutorización, entrenamiento,

asesoramiento entre iguales, consulta y orientación en procesos

educativos y formativos

CE14. Asesorar sobre el uso pedagógico e integración curricular de los medios didácticos

CE15. Analizar, diseñar y evaluar las aplicaciones de las tecnologías de la información y la comunicación

CE16. Identificar planteamientos y problemas educativos, indagar sobre ellos

$\begin{array}{lllllll}2,67 & 1,22 & 2,86 & 1,31 & -1,30 & 98 & 0,19\end{array}$

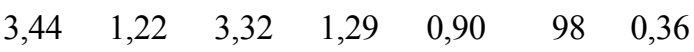

$\begin{array}{lllllll}2,76 & 1,16 & 3,33 & 1,29 & -3,89 & 98 & 0,00\end{array}$

$\begin{array}{lllllll}2,98 & 1,17 & 3,10 & 1,30 & -0,90 & 98 & 0,36\end{array}$

$\begin{array}{lllllll}2,53 & 1,19 & 2,39 & 1,34 & 1,03 & 98 & 0,30\end{array}$

$\begin{array}{lllllll}3,59 & 1,16 & 3,51 & 1,27 & 0,56 & 98 & 0,57\end{array}$

$\begin{array}{lllllll}2,90 & 1,19 & 2,83 & 1,27 & 0,61 & 98 & 0,54\end{array}$

$\begin{array}{lllllll}2,78 & 1,20 & 2,56 & 1,30 & 1,89 & 98 & 0,06\end{array}$

$\begin{array}{lllllll}3,75 & 0,91 & 3,91 & 1,07 & -1,29 & 98 & 0,19\end{array}$

CE17. Diagnóstico de situaciones complejas con especial atención a la $\quad \begin{array}{llllll}3,99 & 1,02 & 3,96 & 1,25 & 0,25 & 98\end{array} \quad 0,80$ diversidad y a la inclusión social 
Tabla 3

Diferenciade mediasen función del curso académico (2014-2015/2015-2016)

Curso académico

Competencias

Diferencias Pre-Test Diferencias Post-Test

\section{Prueba Levene \\ Generales \\ CG4. Capacidad para valorar el impacto social y medioambiental de actuaciones y decisiones en el ámbito educativo y social}

\section{Optar Específicas}

CE7. Diagnosticar las necesidades y posibilidades de desarrollo de las personas

CE8. Conocer y comprender los elementos, procesos y valores de educación y su incidencia en la formación integral

CE9. Diseñar planes, programas, proyectos, propuestas innovadoras de formación, acciones y recursos adaptados a los distintos niveles del sistema educativo CE11. Desarrollar estrategias y técnicas para promover la participación y el aprendizaje a lo largo de la vida CE12. Aplicar y coordinar programas educativos de desarrollo personal, social y profesional

CE14. Conocer y evaluar políticas, instituciones sistemas y organismos educativos

CE15. Conocimiento y aplicación de las herramientas propias del diagnóstico, evaluación y análisis en Pedagogía

CE16. Organizar y gestionar centros, instituciones, servicios y recursos educativos y formativos

CE17. Supervisar planes, programas, centros y profesionales de la educación y la formación

CE19. Aplicar estrategias y técnicas de tutorización, entrenamiento, asesoramiento entre iguales, consulta y orientación en procesos educativos y formativos CE22. Identificar planteamientos y problemas educativos, indagar sobre ellos

\begin{tabular}{|c|c|c|c|c|c|c|c|}
\hline$\mu_{\mathrm{P} 1}$ & $\mu_{\mathrm{P} 2}$ & $t$ & $p$ & $\mu_{\mathrm{P} 1}$ & $\mu_{\mathrm{P} 2}$ & $t$ & $p$ \\
\hline 3,35 & 3,62 & 18,03 & 0,00 & 3,87 & 3,88 & - & - \\
\hline$\mu_{\mathrm{P} 1}$ & $\mu_{\mathrm{P} 2}$ & $t$ & $p$ & $\mu_{\mathrm{P} 1}$ & $\mu_{\mathrm{P} 2}$ & $t$ & $p$ \\
\hline 3,83 & 4,10 & 15,60 & 0,00 & 3,67 & 3,90 & 6,75 & 0,01 \\
\hline 3,72 & 4,08 & 10,29 & 0,00 & 3,98 & 4,28 & - & - \\
\hline 3,37 & 3,65 & 4,43 & 0,03 & 3,38 & 3,58 & - & - \\
\hline 3,45 & 3,79 & 8,486 & 0,00 & 3,52 & 3,40 & - & - \\
\hline 3,33 & 3,73 & 9,10 & 0,00 & 3,31 & 3,40 & - & - \\
\hline 2,43 & 3,04 & 5,90 & 0,01 & 2,71 & 2,99 & - & - \\
\hline 3,20 & 3,75 & 12,46 & 0,00 & 3,23 & 3,36 & - & - \\
\hline 2,43 & 3,13 & 4,611 & 0,03 & 3,67 & 2,97 & - & - \\
\hline 2,62 & $3 ., 5$ & 6,51 & 0,01 & 3,25 & 2,88 & - & - \\
\hline 3,38 & 3,62 & 13,25 & 0,00 & 3,40 & 3,66 & - & - \\
\hline 3,42 & 3,90 & 11,92 & 0,00 & 3,96 & 3,73 & 5,41 & 0,02 \\
\hline
\end{tabular}

Nota: P1: Curso académico 2014-2015. P2: Curso académico 2015-2016.

la percepción que estos tienen de sus competencias, tanto antes como después de la realización de las prácticas académicas. Consultadas las actas de evaluación de los estudiantes, se observa que tanto los que tienen calificaciones finales excelentes como aquellos cuya calificación final es más baja o intermedia manifiestan, en general, una percepción positiva respecto a la adquisición de competencias profesionales, tanto antes como después de realizar las prácticas externas. No obstante, los resultados ponen de manifiesto (tabla 4) que en la aplicación pre-test se obtienen diferencias significativas en la competencia relacionada con la organización y gestión de centros e instituciones(CE16), a favor de los estudiantes cuya calificación media es de Sobresaliente (9-10 sobre 10). En la aplicación post-test se obtienen diferencias en dos de las competencias examinadas, la primera de ellas relacionada con la capacidad de aprendizaje autónomo y la responsabilidad del estudiante (CG2), entre los estudiantes de Matrícula de Honor (10 MH sobre 10) con respecto a los estudiantes 
cuya calificación es Notable (7-8 sobre 10) o Aprobado (5-6 sobre 10); y la segunda, vinculada al conocimiento yaplicación de herramientas propias del diagnóstico, evaluación y análisis de la Pedagogía (CE15) entre los estudiantes de Sobresaliente y Aprobado con respecto a los estudiantes de Notable.

Tal como se refleja en la tabla 4 , se confirma que aquellos alumnos cuyo rendimiento en la asignatura es menor son los que reconocen haber adquirido en menor porcentaje las competencias profesionales examinadas, necesarias para el desempeño de las prácticas.

\section{Diferencias en las valoraciones en función de la Mención cursada}

A partir de las puntuaciones medias totales, quienes mejor valoran la adquisición de competencias tanto en la aplicación pre-test como en la post-test, son los estudiantes que cursan la mención de Orientación Educativa y Asesoramiento $(\mu=4,40)$ seguidos de los que cursan la modalidad sin mención $(\mu=4,30)$ y por último los de la mención de Formación y Gestión de calidad $(\mu=4,23)$.

En el pre-test se encontraron diferencias significativas en siete de las veintitrés competencias. Tres de ellas indican que los alumnos que cursan la mención de Orientación Educativa valoraban por encima del resto de estudiantes la adquisición de las competencias: Capacidad de autoconocimiento para el desarrollo personal y profesional (CG6), aplicar estrategias y técnicas de tutorización, entrenamiento, asesoramiento entre iguales, consulta y orientación en procesos educativos y formativos (CE13) y diagnóstico de situaciones complejas con especial atención a la diversidad y a la inclusión social (CE23); mientras que las competencias:Conocer $\mathrm{y}$ evaluar políticas, instituciones sistemas y organismos educativos (CE14), organizar y gestionar centros, instituciones, servicios y recursos educativos y formativos (CE16), supervisar planes, programas, centros y profesionales de la educación y la formación (CE17) y desarrollar procesos y modelos de gestión de calidad de la educación y la formación (CE18), las valoran con puntuaciones más altas los estudiantes de la mención Formación y Gestión antes de realizar las prácticas externas (tabla 5).

En el pos-test sin embargo, se comprobó que los estudiantes de la Mención Orientación Educativa otorgaron puntuaciones más elevadas en la adquisición de las competencias:Desarrollar y coordinar intervenciones educativas con personas o grupos, con necesidades específicas, en situaciones de riesgo, de desigualdad o discriminación (CE13) y diagnóstico de situaciones complejas con especial atención a la diversidad y a la inclusión social (CE23) en comparación con el resto de estudiantes, mientras que los estudiantes de la mención Formación y Gestión otorgan puntuaciones más elevadas, existiendo diferencias significativas respecto al resto de estudiantes, en las competencias: Organizar y gestionar centros, instituciones, servicios y recursos educativos y formativos(CE16), supervisar planes, programas, centros y profesionales de la educación y la formación (CE17) y desarrollar procesos y modelos de gestión de calidad de la educación y la formación (CE18).

Tabla 4

Diferencia de medias en función del rendimiento académico

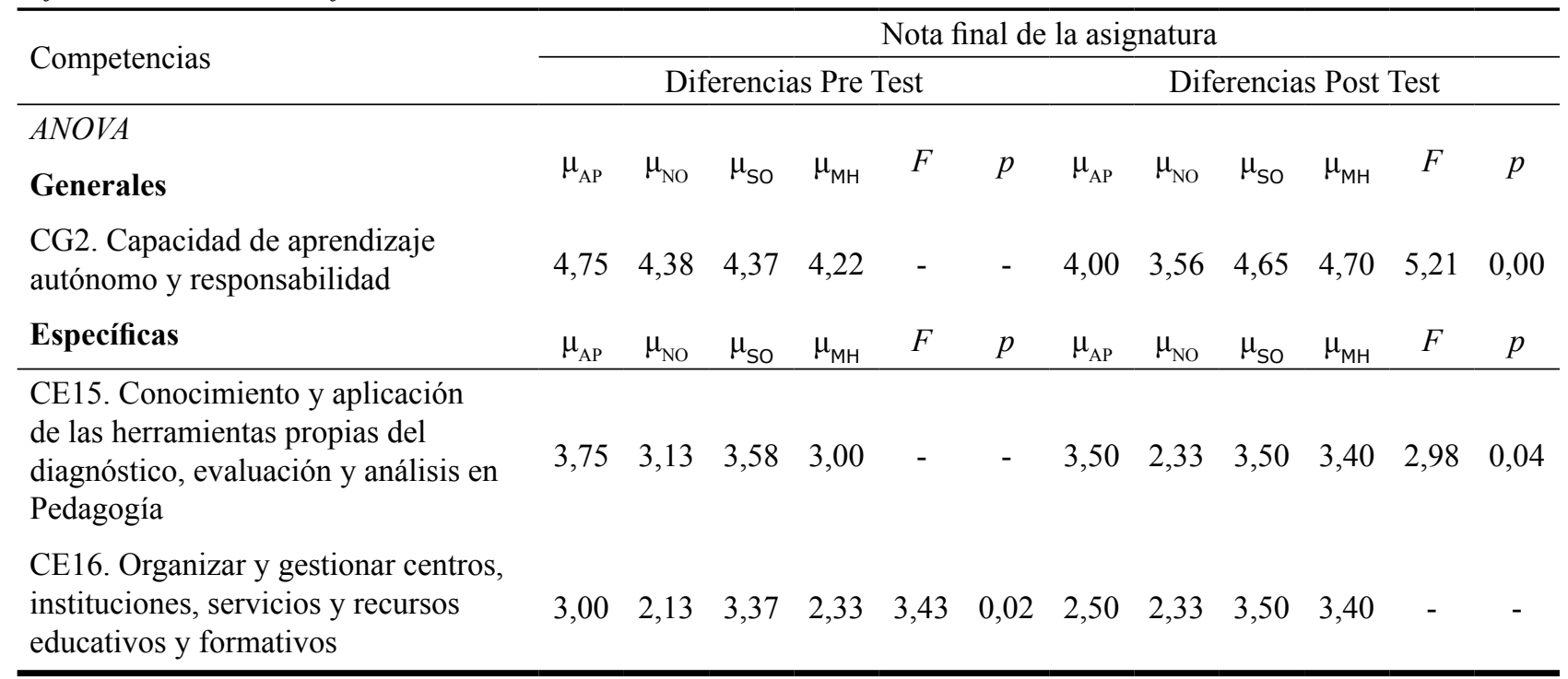


Tabla 5

Diferencia de medias en función de la mención cursada

\begin{tabular}{|c|c|c|c|c|c|c|c|c|c|c|}
\hline \multirow{3}{*}{$\begin{array}{l}\text { Competencias } \\
A N O V A \\
\text { Generales }\end{array}$} & \multicolumn{10}{|c|}{ Mención } \\
\hline & \multicolumn{5}{|c|}{ Diferencias Pre Test } & \multicolumn{5}{|c|}{ Diferencias Post Test } \\
\hline & $\mu_{\mathrm{O}}$ & $\mu_{\mathrm{FG}}$ & $\mu_{S M}$ & $F$ & $p$ & $\mu_{\mathrm{o}}$ & $\mu_{\mathrm{FG}}$ & $\mu_{S M}$ & $F$ & $p$ \\
\hline $\begin{array}{l}\text { CG6. Capacidad de } \\
\text { autoconocimiento para el desarrollo } \\
\text { personal y profesional }\end{array}$ & 4,34 & 3,64 & 4,23 & 5,41 & 0,00 & 4,40 & 4,08 & 4,30 & - & - \\
\hline Específicas & $\mu_{\mathrm{O}}$ & $\mu_{\mathrm{FG}}$ & $\mu_{S M}$ & $F$ & $p$ & $\mu_{\mathrm{O}}$ & $\mu_{\mathrm{FG}}$ & $\mu_{S M}$ & $F$ & $p$ \\
\hline $\begin{array}{l}\text { CE13. Desarrollar y coordinar } \\
\text { intervenciones educativas con } \\
\text { personas o grupos, con necesidades } \\
\text { específicas, en situaciones } \\
\text { de riesgo, de desigualdad o } \\
\text { discriminación }\end{array}$ & 4,12 & 3,29 & 4,00 & 3,94 & 0,02 & 4,15 & 3,00 & 3,94 & 4,52 & 0,01 \\
\hline $\begin{array}{l}\text { CE14. Conocer y evaluar políticas, } \\
\text { instituciones sistemas y organismos } \\
\text { educativos }\end{array}$ & 2,44 & 3,93 & 2,69 & 9,50 & 0,00 & 2,72 & 3,54 & 3,08 & - & - \\
\hline $\begin{array}{l}\text { CE16. Organizar y gestionar } \\
\text { centros, instituciones, servicios y } \\
\text { recursos educativos y formativos }\end{array}$ & 2,54 & 3,79 & 2,72 & 7,32 & 0,00 & 3,55 & 3,77 & 2,82 & 4,35 & 0,01 \\
\hline $\begin{array}{l}\text { CE17. Supervisar planes, } \\
\text { programas, centros y profesionales } \\
\text { de la educación y la formación }\end{array}$ & 2,81 & 3,50 & 2,85 & - & - & 3,09 & 3,92 & 2,79 & 3,72 & 0,02 \\
\hline $\begin{array}{l}\text { CE18. Desarrollar procesos y } \\
\text { modelos de gestión de calidad de la } \\
\text { educación y la formación }\end{array}$ & 2,32 & 3,36 & 2,51 & 4,72 & 0,01 & 2,25 & 3,23 & 2,30 & 3,03 & 0,05 \\
\hline $\begin{array}{l}\text { CE23. Diagnóstico de situaciones } \\
\text { complejas con especial atención a la } \\
\text { diversidad y a la inclusión social }\end{array}$ & 4,03 & 3,14 & 3,95 & 3,53 & 0,03 & 4,17 & 2,92 & 4,03 & 5,75 & 0,00 \\
\hline
\end{tabular}

\section{Diferencias en las valoraciones en función de la titularidad del centro}

Las puntuaciones más elevadas las conceden aquellos alumnos que han realizado sus prácticas en centros de titularidad concertada $(\mu=4,73)$ y las puntuaciones más bajas proceden de los que las han realizado en centros de titularidad privada $(\mu=3,81)$.

En relación a la titularidad del centro receptor de los alumnos en prácticas (pública, privada o concertada), se comprobaron diferencias estadísticamente significativas (tabla 6) en la aplicación pre-test en las competencias: Conocer y evaluar políticas, instituciones sistemas y organismos educativos (CE14), organizar y gestionar centros, instituciones, servicios y recursos educativos y formativos (CE16) y diagnóstico de situaciones complejas con especial atención a la diversidad y a la inclusión social (CE17), a favor de los que realizarían sus prácticas en centros de titularidad pública, y también en los que iban a realizar prácticas en centros de titularidad concertada en las competencias: Analizar, diseñar y evaluar las aplicaciones de las tecnologías de la información y la comunicación(CE15), desarrollar procesos y modelos de gestión de calidad de la educación y la formación (CE18), aplicar estrategias y técnicas de tutorización, entrenamiento, asesoramiento entre iguales, consulta y orientación en procesos educativos y formativos (CE19), asesorar sobre el uso pedagógico e integración curricular de los medios didácticos (CE20), analizar, diseñar y evaluar las aplicaciones de las tecnologías de la información y la comunicación (CE21), identificar planteamientos y problemas educativos, indagar sobre ellos (CE22) y diagnóstico de situaciones complejas con especial atención a la diversidad y a la inclusión social (CE23). 
En la aplicación post-test, se verificaron dos de las diferencias observadas en la aplicación pre-test, en la adquisición de competencias, en función de los centros de titularidad pública: Conocer y evaluar políticas, instituciones sistemas y organismos educativos (CE14) y organizar y gestionar centros, instituciones, servicios y recursos educativos y formativos (CE16); y en los de titularidad concertada, en las competencias:Capacidad para el trabajo en equipo (CG1), diagnosticar las necesidades y posibilidades de desarrollo de las personas (CE7), conocer y comprender los elementos, procesos y valores de educación y su incidencia en la formación integral (CE8), conocimiento y aplicación de las herramientas propias del diagnóstico, evaluación y análisis en Pedagogía (CE15), supervisar planes, programas, centros y profesionales de la educación y la formación (CE17), asesorar sobre el uso pedagógico e integración curricular de los medios didácticos (CE20) y analizar, diseñar y evaluar las aplicaciones de las tecnologías de la información y la comunicación (CE21).

\section{Discusión y conclusiones}

En lo que a la adquisición de competencias profesionales se refiere, es habitual en otros estudios (Lasa, 2006; Serrate et al.,2016) encontrar en los alumnosuna alta deseabilidad por adquirir multitud de conocimientos y competencias durante su periodo de aprendizaje en un centro o institución profesional. Este hecho se debe a la motivación intrínseca por conocer su ámbito profesional de cerca, salir de los muros de la Universidad y aprender desde y en la propia práctica. Sin embargo, una vez finalizado el periodo de experimentación profesional, concluida la fase de aprendizaje en la institución de referencia, la evaluación suele ser, en la mayoría de los casos, másajustada a la realidad que han podido observar y sobre la que han podido actuar. Es ahí donde ejerce influencia, en la adquisición de competencias,el tipo de centro o institución en el que se han realizado las prácticas, la mención cursada, los conocimientos adquiridos previamente, la implicación personal del alumno, etc.

A partir de los resultados del presente estudio, podemos afirmar que la hipótesis de partida se comprueba parcialmente, puesen general la percepción respecto a la adquisición de competencias no aumenta tras el periodo de prácticas, al contrario, en algunas, disminuye considerablemente, confirmándose que no se han cumplido las expectativas de los estudiantes en este sentido. Nos referimos a competencias vinculadas a la capacidad emprendedora de los estudiantes, al diagnóstico de necesidades y posibilidades de desarrollo, a la evaluación de planes, programas y proyectos y al desarrollo de estrategias que promuevan la participación. La heterogeneidad de los centros colaboradores externos a la Universidad, el momento en el que el estudiante se incorpora a la rutina de las instituciones, las características variantes del trabajo desarrollado o los usuarios con los que trabajan, condicionanel desarrollo de aquellas competencias que, en un principio, pensaban y deseaban desarrollar.

No obstante, los resultados obtenidos muestran que, al contrario, existen determinadas competenciasque los estudiantes consideran que no van a desarrollar tanto como posteriormente afirman haber adquirido. Nos referimos a aquellas competencias relativas a la capacidad de los alumnos para valorar el impacto social y medioambiental de sus actuaciones y decisiones en al ámbito educativo y social, el tener la oportunidad de conocer y comprender los elementos, procesos y valores de educación y su incidencia en la formación integral y el poder organizar y gestionar centros, instituciones, servicios y recursos educativos y formativos. Aunque existen estudios (Armengol et al., 2011; Martínez \& Viader, 2008) en los que se pone de manifiesto que la escasa duración en el tiempo del Prácticum, en las titulaciones de educación, no favorecen la posibilidad de una inmersión en la realidad práctica (en el caso de la Universidad de Salamanca, 240 horas en tres meses), se puede comprobar que este tiempo sí permite, en determinados casos,el desarrollo de competencias vinculadas a la configuración global de las actuaciones del pedagogo, asociadas tanto al saber cómo al saber hacer.

No existen investigaciones en las que se compruebe que el rendimiento académico de los estudiantes influye significativamente en la adquisición de competencias profesionales.El supuesto de que los estudiantes con mejor rendimiento académico en el resto de materias que componen los estudios de Pedagogía, valoran la adquisición de competencias prácticas mejor que el resto de estudiantes, se confirma parcialmente. Los datos obtenidos en este estudio muestran que la adquisición de determinadas competencias es valorada con mayores puntuaciones por alumnoscuyo rendimiento es sobresaliente, tal es el caso de la gestión de centros e instituciones o la capacidad de aprendizaje autónomo $y$ de responsabilidad, aspectos en los que se implica la iniciativa personal del estudiante, tanto hacia el aprendizaje como hacia la realización de tareas. Estos mismos alumnos, al igual que aquellos cuyo rendimiento ronda el aprobado, también valoran de una manera muy positiva la capacidad para aplicar las herramientas propias del diagnóstico, la evaluación y el análisis desde al punto de vida pedagógico. La variable rendimiento académico, por lo tanto, no ayuda a discriminar resultados relevantes para la investigación sobre la adquisición de competencias prácticas, aun así, permite confirmar que aquellos estudiantes que tienen mejores calificaciones académicas, valoran mejor las competencias relacionadas con trabajo autónomo y responsabilidad personal hacia la profesión, adquiridas a partir de un periodo de prácticas en instituciones profesionales. 
Tabla 6

Diferencia de medias en función de la titularidad del centro de prácticas

\begin{tabular}{|c|c|c|c|c|c|c|c|c|c|c|}
\hline \multirow{3}{*}{$\begin{array}{l}\text { Competencias } \\
\text { ANOVA }\end{array}$} & \multicolumn{10}{|c|}{ Titularidad del centro } \\
\hline & \multicolumn{5}{|c|}{ Diferencias Pre Test } & \multicolumn{5}{|c|}{ Diferencias Post Test } \\
\hline & $\mu_{\mathrm{PU}}$ & $\mu_{\mathrm{C}}$ & $\mu_{\mathrm{PR}}$ & $F$ & $p$ & $\mu_{\mathrm{PU}}$ & $\mu_{\mathrm{C}}$ & $\mu_{\mathrm{PR}}$ & $F$ & $p$ \\
\hline \multicolumn{11}{|l|}{ Generales } \\
\hline $\begin{array}{l}\text { CG1. Capacidad para el trabajo en } \\
\text { equipo }\end{array}$ & 3,98 & 4,08 & 3,89 & - & - & 4,13 & 4,44 & 3,60 & 7,16 & 0,00 \\
\hline Específicas & $\mu_{\mathrm{PU}}$ & $\mu_{\mathrm{C}}$ & $\mu_{\mathrm{PR}}$ & $F$ & $p$ & $\mu_{\mathrm{PU}}$ & $\mu_{\mathrm{C}}$ & $\mu_{\mathrm{PR}}$ & $F$ & $p$ \\
\hline $\begin{array}{l}\text { CE7. Diagnosticar las necesidades } \\
\text { y posibilidades de desarrollo de las } \\
\text { personas }\end{array}$ & 3,87 & 4,29 & 3,93 & - & - & 3,68 & 4,30 & 3,57 & 4,47 & 0,01 \\
\hline $\begin{array}{l}\text { CE8. Conocer y comprender los } \\
\text { elementos, procesos y valores de } \\
\text { educación y su incidencia en la } \\
\text { formación integral }\end{array}$ & 3,85 & 4,13 & 3,74 & - & - & 4,08 & 4,59 & 3,97 & 5,61 & 0,00 \\
\hline $\begin{array}{l}\text { CE14. Conocer y evaluar políticas, } \\
\text { instituciones sistemas y organismos } \\
\text { educativos }\end{array}$ & 2,96 & 2,75 & 2,22 & 3,267 & 0,04 & 3,05 & 2,96 & 2,33 & 3,47 & 0,03 \\
\hline $\begin{array}{l}\text { CE15. Conocimiento y aplicación de las } \\
\text { herramientas propias del diagnóstico, } \\
\text { evaluación y análisis en Pedagogía }\end{array}$ & 3,48 & 4,25 & 2,78 & 11,291 & 0,00 & 3,05 & 4,19 & 3,00 & 9,54 & 0,00 \\
\hline $\begin{array}{l}\text { CE16. Organizar y gestionar centros, } \\
\text { instituciones, servicios y recursos } \\
\text { educativos y formativos }\end{array}$ & 2,93 & 2,75 & 2,52 & 1,08 & 0,34 & 3,43 & 3,44 & 2,77 & 3,02 & 0,05 \\
\hline $\begin{array}{l}\text { CE17. Supervisar planes, programas, } \\
\text { centros y profesionales de la educación } \\
\text { y la formación }\end{array}$ & 3,13 & 3,00 & 2,70 & 1,11 & 0,33 & 3,08 & 3,44 & 2,53 & 3,80 & 0,02 \\
\hline $\begin{array}{l}\text { CE18. Desarrollar procesos y modelos } \\
\text { de gestión de calidad de la educación y } \\
\text { la formación }\end{array}$ & 2,63 & 2,88 & 2,07 & 3,30 & 0,04 & 2,52 & 2,67 & 2,10 & - & - \\
\hline $\begin{array}{l}\text { CE19. Aplicar estrategias y técnicas } \\
\text { de tutorización, entrenamiento, } \\
\text { asesoramiento entre iguales, consulta } \\
\text { y orientación en procesos educativos y } \\
\text { formativos }\end{array}$ & 3,59 & 4,17 & 3,11 & 5,68 & 0,00 & 3,58 & 3,93 & 3,27 & - & - \\
\hline $\begin{array}{l}\text { CE20. Asesorar sobre el uso pedagógico } \\
\text { e integración curricular de los medios } \\
\text { didácticos }\end{array}$ & 2,89 & 3,25 & 2,67 & 1,57 & 0,21 & 2,83 & 3,37 & 2,43 & 4,15 & 0,01 \\
\hline $\begin{array}{l}\text { CE21. Analizar, diseñar y evaluar las } \\
\text { aplicaciones de las tecnologías de la } \\
\text { información y la comunicación }\end{array}$ & 2,78 & 3,29 & 2,33 & 4,326 & 0,01 & 2,40 & 3,15 & 2,40 & 3,78 & 0,02 \\
\hline $\begin{array}{l}\text { CE22. Identificar planteamientos y } \\
\text { problemas educativos, indagar sobre } \\
\text { ellos }\end{array}$ & 3,87 & 4,00 & 3,37 & 3,85 & 0,02 & 3,78 & 4,19 & 3,60 & - & - \\
\hline $\begin{array}{l}\text { CE23. Diagnóstico de situaciones } \\
\text { complejas con especial atención a la } \\
\text { diversidad y a la inclusión social }\end{array}$ & 3,96 & 4,46 & 3,63 & 4,49 & 0,01 & 3,85 & 4,22 & 3,80 & - & - \\
\hline
\end{tabular}


La mención o especialización de los estudios cursados es indicativo de una diferente adquisición de competencias. Los que cursan la rama de Orientación Educativa, asumen adquirir competencias más vinculadas al desarrollo y coordinación de intervenciones educativas con personas o grupos, con necesidades especificas, en situaciones de riesgo, de desigualdad o de discriminación $\mathrm{y}$ al diagnóstico de situaciones complejas con especial atención a la diversidad y a la inclusión social, competencias en sí mismas propias del trabajo de un orientador, tanto en un centro escolar como en una intuición que trabaja desde una perspectiva pedagógica más social. Los resultados muestran que los estudiantes especializados en el área de la Formación y la Gestión perciben adquirir competencias relacionadas con organización y gestión de centros, instituciones, servicios y recursos educativos y formativos, la supervisión de planes, programas, centros y profesionales de la educación y el desarrollo de procesos y modelos de gestión de calidad de la educación y la formación, competencias todas ellas vinculadas al trabajo que ejerce un profesional de la pedagogía dentro de los equipos de planificación educativao en instituciones de coordinación pedagógica, así como en espacios de gestión de recursos humanos (Serrate et al.,2016; Vilà et al., 2015).

Coincidimos con autores como Aneas yReguant (2015) y Antón y Hevia (2011)en que elementos como la organización o distribución del trabajo no es igual en función de la institución receptora o de su titularidad. Así, los centros de titularidad pública permiten adquirir competencias más vinculadas al conocimiento y evaluación de políticas, instituciones, sistemas y organismos educativos, así como organizar y gestionar centros $e$ instituciones; mientras que los centros de titularidad concertada, la adquisición de competencias vinculadas al trabajo en equipo, al diagnóstico de las necesidad y posibilidades personales de los sujetos con los que se trabaja, al conocimiento y la comprensión de forma integral de los procesos, elemento y valores educativos, al conocimiento y aplicación de las herramientas propias del diagnóstico y evaluación pedagógica, a la supervisión de planes y programas, al asesoramiento sobre el uso pedagógico e integración curricular de medios didácticos y al análisis, diseño y evaluación de aplicaciones de las tecnologías de la información y la comunicación.

No cabe duda, a partir de lo expuesto hasta el momento, de que el periodo de prácticas externas es un momento crucial en la formación, más aún cuando este es único a lo largo de los cuatro años de estudios (Vianello, Pérez, \&Pacios, 2013). Estamos de acuerdo con Aneas, Vilà yAlòs (2015) en que permite tomar conciencia sobre la cultura profesional y comprobar la especialización del trabajo en función del espacio concreto, además de conocer un contexto de referencia donde comprender los fenómenos que tienen lugar en la realidad profesional, desde una perspectiva educativa, formativay social. También, ofrece la posibilidad de poner en práctica experiencias formativas diferentes a las diseñadas por la Universidad, en donde los alumnos ponen a prueba tanto los conocimientos previos adquiridos como su capacidad de trabajo autónomo y responsable. Además,permiten crear una identidad profesional individual y colectiva, de tal forma que el estudiante se conoce más así mismo en una faceta hasta el momento desconocida y le permite identificarse como miembro de un grupo. Y, por último, el espacio de prácticas ofrece al estudiante identificar estrategias para acceder al mercado laboral, a partir del intercambio con otros profesionales que se encuentran en espacios de empleo.

Por último, señalar que se han tenido en cuenta los aspectos éticos, propios de los estudios de estas características, en la obtención y tratamiento de la información.

Las limitaciones del trabajo, se encuentran fundamentalmente en el instrumento de medida basado en la auto-percepción delos estudiantes. Para futuros estudios sería aconsejable combinar la obtención de resultados con algún otro instrumento de medida, al igual que introducir datos respecto a una posible evaluación externa, como puede ser la valoración de los tutores profesionales para obtener medidas más consistentes que atiendan a la relevancia de las prácticas para el desarrollo profesional de los estudiantes de Pedagogía.

\section{Referencias}

Aneas, A., \&Reguant, M. (2015). Evaluación de los resultados de aprendizaje. El caso del Prácticum del grado de pedagogía de la universidad de Barcelona. In AIDIPE (Ed.), Investigar con y para la sociedad (Vol. 2, pp. 1071-1081). Cádiz: Bubok.

Aneas,A., \&Vilà, R (2015). ¿Hay cambios en la percepción que tiene el alumnado sobre su competencia tras haber desarrollado su Prácticum? El caso del Prácticum del grado de Pedagogía de la Universidad de Barcelona. In AIDIPE (Ed.), Investigar con y para la sociedad (Vol. 2, pp. 1073-1084). Cádiz: Bubok.

Aneas, A., Vilà, R., \&Alòs, M. (2015). La vinculación de las prácticas externas y el trabajo final de grado. Percepciones del alumnado sobre su desarrollo competencial. In UNIVEST (Ed.), Los retos de mejorar la evaluación (pp. 402408). Girona: Dipòsit Digital de la Universitat de Girona. 
Antón, X. \& Hevia, I. (2011). El Prácticum de la Licenciatura de Pedagogía: estudio empírico desde la perspectiva del alumnado. Revista de Educación, 354, 209-236. Recuperado en 15 mayo 2014, de http://www.revistaeducacion.mec. es/re354/re354_09.pdf

Armengol, C., Castro, D., Jariot, M., Massot, M., \& Sala, J. (2011). El Prácticum en el Espacio Europeo de Educación Superior (EEES): mapa de competencias del profesional de la educación. Revista de Educación, 354, 71-98. Recuperado en 24 abril 2014, de http://www.univnova.org/documentos/492.pdf

Bretones, A. (2013). El Prácticum de magisterio en educación primaria: una mirada retrospectiva. Revista Complutense de Educación, 24(2), 443-471. Recuperado en 22 mayo 2015, de http://revistas.ucm.es/index.php/RCED/article/ view/42088

Cabrerizo, J., Rubio, M.J., \& Castillo, S. (2010). El Prácticum en los grados de pedagogía, de magisterio y de educación social. Madrid: Pearson Educación.

Cabrerizo, J., Rubio, M.J., Castillo, S., \& Cabrerizo, A. (2011). El Prácticum del máster en formación del profesorado. Madrid: Pearson Educación.

Campbell, D.T., \&Standley, J.C. (1963). Experimental and Quasi-Experimental deigns for research. Chicago, IL: Rand-McNally.

Casillas, S., González, M., \& Serrate, S. (2015). Percepción de los estudiantes del Grado en Pedagogía y Educación Social sobre la organización, utilidad y formación del Prácticum. Enseñanza \& Teaching, 33(2), 171-190. doi: 10.14201/ et2015332171190.

Cid, A., Pérez, A., \& Sarmiento, J.A. (2011). La tutoría en el Prácticum. Revisión de la literatura. Revista de Educación, 354, 127-154. Recuperado en 10 junio 2012, de http://www.revistaeducacion.educacion.es/re354/re354_06.pdf

De Miguel, M. (2005). Modalidades de enseñanza centradas en el desarrollo de competencias. Orientaciones para promover el cambio metodológico en el marco del espacio europeo de educación superior. Oviedo: Ediciones Universidad de Oviedo.

Díaz, V. (2012). Ventajas e inconvenientes de la encuesta por internet. Papers, 97(1), 193-223. doi: http://dx.doi. org/10.5565/rev/papers/v97n1.71

González, N. (2006). Evaluación y mejora del Prácticum en las titulaciones de Ciencias de la Educación de la UPV/EHU. Revista de Psicodidáctica, 11(1), 145-158.

González, M., \&Fuertes, E.J. (2011). El Prácticum en el aprendizaje de la profesión docente. Revista de Educación, 354, 47-70. Recuperado en 27 mayo 2014, de http://www.revistaeducacion.educacion.es/re354/re354_03.pdf

Latorre, M.J., \&Blanco, F.J. (2009). El conocimiento de las percepciones y opiniones de los estudiantes y tutores de los centros externos sobre la incidencia del Prácticum en la formación de los psicopedagogos. In M. Raposo, M.E. Martínez, L. Lodeiro, J.C. Fernández, \& A. Pérez (Coords.), El Prácticum más allá del empleo. Formación vs. Training (pp. 1279-1293). Pontevedra: Imprenta universitaria.

Lasa, A. (2006). El Prácticum en la Facultad de Psicología de la UNED: Resultados de la evaluación de su experiencia piloto en el itinerario de la Psicología Clínica. Acción Psicológica, 4(1), 25-36.

Latorre, M.J., \&Blanco, F.J. (2011). El Prácticum como espacio de aprendizaje profesional para docentes en formación. Revista de Docencia Universitaria, 9(2), 35-54. Recuperado en 14 enero 2014, de http://red-u.net/redu/files/journals/1/articles/269/public/269-532-3-PB.pdf

Martínez, M., \&Viader, M. (2008). Reflexiones sobre aprendizaje y docencia en el actual contexto universitario. La promoción de equipos docentes. Revista de Educación, número extraordinario 2008, 213-234. Recuperado en 15 febrero 2015, de http://www.revistaeducacion.mec.es/re2008/re2008_09.pdf

Mendoza, M., \&Covarrubias, C.G. (2014). Valoración del Prácticum de los grados del magisterio desde la perspectiva de sus estudiantes. Revista Electrónica Educare,18(3), 111-142. doi: http://dx.doi.org/10.15359/ree

Real Decreto 1707/2011 (2011, 18 de noviembre). Regulación de las prácticas académicas externas de los estudiantes universitarios. Madrid: Presidencia de España. 
Rego, L., Rial, A.F., \&Barreira, E.M. (2015). La formación en alternancia en la Universidad y en los ciclos formativos: aportaciones desde dos investigaciones realizadas en Galicia. Educar, 51(2), 349-371. Recuperado en 23 octubre 2016, de http://www.raco.cat/index.php/Educar/article/view/295239

Rial, A.F., \&Barreira, E.M. (2012). El Prácticum de la Titulación de Pedagogía desde el punto de vista profesional. Enseñanza \&Teaching, 30(2), 153-174.

Rodicio, M.L., \&Iglesias, M. (2011). La formación en competencias a través del Prácticum: un estudio piloto. Revista de Educación, 354, 99-124. Recuperado en 15 diciembre 2012, de http://repositorio.minedu.gob.pe/handle/123456789/988

Rodríguez, A.J., Calmaestra, J., \&Maestre, M. (2015). Desarrollo de competencias en el Prácticum de maestros: ABP y Coaching Multidimensional. Profesorado. Revista de currículum y formación del profesorado, 19(1), 414-434. Recuperado en 21 enero 2016, de http://recyt.fecyt.es/index.php/profesorado/article/view/41049/23333

Serrate, S., Casillas, S., \& Cabezas, M. (2016). Estudio de los criterios para la selección de centros de prácticas de calidad. Una propuesta de evaluación para mejorar la formación de los pedagogos. Estudios pedagógicos, 42(3), 369-389. doi: http://dx.doi.org/10.4067/S0718-07052016000400020.

Vianello, M., Pérez, B., \&Pacios, A.R. (2013). El Prácticum del grado en Información y Documentación: la experiencia en la UC3M. Anales de Documentación, 16(2). doi: http://dx.doi.org/10.6018/analesdoc.16.2.180381

Vilà, R., Aneas, A.,\&Rajadell, N. (2015). La evaluación de competencias del alumnado en las Prácticas Externas. La perspectiva de todos los agentes implicados en las Prácticas Externas del grado de Pedagogía de la Universidad de Barcelona. Procedia, 196, 226-232.

Zabalza, M.A. (2011). El Prácticum en la formación universitaria: estado de la cuestión. Revista de Educación, 354, 2143. Recuperado en 25 enero 2014, de http://www.revistaeducacion.educacion.es/re354/re354_02.pdf

Recebido: $28 / 03 / 17$

Aceito: $29 / 08 / 18$

Sobre os autores:

Sonia Casillas Martín é Licenciada en Pedagogía, Licenciada en Psicopedagogía y Doctora en Pedagogía. Profesora en el Departamento de Didáctica, Organización y Métodos de Investigación en la Facultad de Educación de la Universidad de Salamanca.

Marcos Cabezas Gonzálezé Licenciado en Pedagogía, Diplomado en Educación Social y Doctor en Pedagogía. Profesor en el Departamento de Didáctica, Organización y Métodos de Investigación de la Facultad de Educación de la Universidad de Salamanca.

Sara Serrate González é Graduada en Educación Social, Licenciada en Psicopedagogía y Doctora en Psicopedagogía. Profesora en el Departamento de Teoría e Historia de la Educación de la Facultad de Educación de la Universidad de Salamanca. 